\title{
Remote magnetic versus manual catheters: evaluation of ablation effect in atrial fibrillation by myocardial marker levels
}

\author{
Eivind Solheim • Morten Kristian Off • Per Ivar Hoff • \\ Alessandro De Bortoli • Peter Schuster • \\ Ole-Jørgen Ohm • Jian Chen
}

Received: 30 September 2010 / Accepted: 9 March 2011 /Published online: 8 April 2011

(C) The Author(s) 2011. This article is published with open access at Springerlink.com

\begin{abstract}
Background A remote magnetic navigation (MN) system is available for radiofrequency ablation of atrial fibrillation (AF), challenging the conventional manual ablation technique. The myocardial markers were measured to compare the effects of the two types of MN catheters with those of a manual-irrigated catheter in AF ablation.

Methods AF patients underwent an ablation procedure using either a conventional manual-irrigated catheter (CIR, $n=65$ ) or an MN system utilizing either an irrigated (RMI, $n=23$ ) or non-irrigated catheter (RMN, $n=26$ ). Levels of troponin $\mathrm{T}(\mathrm{TnT})$ and the cardiac isoform of creatin kinase (CKMB) were measured before and after ablation.

Results Mean procedure times and total ablation times were longer employing the remote magnetic system. In all groups, there were pronounced increases in markers of myocardial injury after ablation, demonstrating a significant correlation between total ablation time and post-ablation levels of TnT and CKMB (CIR $r=0.61$ and 0.53, $p<0.001$; RMI $r=0.74$ and $0.73, p<0.001$; and RMN $r=0.51$ and 0.59 , $p<0.01)$. Time-corrected release of TnT was significantly higher in the CIR group than in the other groups. Of the
\end{abstract}

E. Solheim • M. K. Off • P. I. Hoff • A. De Bortoli • P. Schuster • O.-J. Ohm • J. Chen

Institute of Medicine, University of Bergen,

Bergen, Norway

E. Solheim $(\bowtie) \cdot$ M. K. Off $\cdot$ P. I. Hoff • A. De Bortoli •

P. Schuster $\cdot$ O.-J. Ohm $\cdot$ J. Chen

Department of Heart Disease, Haukeland University Hospital,

5021 Bergen, Norway

e-mail: eivind.solheim@helse-bergen.no patients, 59.6\% were free from $\mathrm{AF}$ at follow-up (12.2 \pm 5.4 months) and there were no differences in success rate between the three groups.

Conclusions Remote magnetic catheters may create more discrete and predictable ablation lesions measured by myocardial enzymes and may require longer total ablation time to reach the procedural endpoints. Remote magnetic non-irrigated catheters do not appear to be inferior to magnetic irrigated catheters in terms of myocardial enzyme release and clinical outcome.

Keywords Atrial fibrillation - Ablation · Myocardial markers $\cdot$ Magnetic navigation $\cdot$ Irrigated catheter

\section{Background}

Radiofrequency ablation (RFA) has evolved as the treatment of choice for drug-resistant atrial fibrillation (AF). The electrical isolation of the pulmonary veins (PVs) is regarded as one endpoint of ablation [1, 2], but more extensive approaches, such as linear- or complexfractionated atrial electrogram (CFAE) ablation, may also be employed. A remote magnetic navigation (MN) system is available and has been shown to be feasible in treating AF $[1,3]$. A soft RFA catheter with a magnet can be guided and positioned by directional magnetic fields. An irrigated RFA catheter has been shown to be capable of enlarging the size and improving the transmurality of ablation lesions using manual catheters [4], but no equivalent data are available for the MN system. A good tissue contact and positional stability are believed to be some of the advantages of the MN system, but it is not known whether $\mathrm{AF}$ ablation using $\mathrm{MN}$ causes similar levels of myocardial 
injury as manual ablation. RFA induces local thermal damage in the heart [5] and previous studies have shown that traditional myocardial markers such as Troponin $\mathrm{T}(\mathrm{TnT})$ and creatine kinase's cardiac isoenzyme MB (CKMB) are sensitive measures of myocardial injury [6-11]. Most studies have been performed when only limited ablation has been applied.

In this study, we sought to determine the relative impacts of the different catheters by measuring levels of myocardial markers $\mathrm{TnT}$ and $\mathrm{CKMB}$ before and after $\mathrm{AF}$ ablation using standard irrigated catheters and both irrigated and non-irrigated $\mathrm{MN}$ catheters.

\section{Methods}

\subsection{Patient selection}

One hundred and fourteen highly symptomatic patients with drug-resistant, paroxysmal or persistent $\mathrm{AF}$ referred for RFA treatment were enrolled. Demographic and clinical data were collected prior to the procedure (Table 1). All patients were given oral anticoagulants for at least 1 month before the procedure. Patients underwent RFA either using a conventional manual-irrigated catheter (CIR group, $n=65$ ) or a remote magnetic navigated ablation procedure that employed irrigated (RMI group, $n=23$ ) or non-irrigated (RMN group, $n=26$ ) catheters, based on the operator's decision. We excluded patients with renal failure, recent myocardial ischemia or clinical signs of infection. All patients provided informed consent. The study was performed in accordance with the Declaration of Helsinki, and was approved by the local ethics committee.

\subsection{Ablation procedure}

Patients underwent electrophysiological study and RFA in a fasting, sedated state. Vascular access was obtained under local anesthesia through the right and left femoral veins. In all patients, a 7F 20-pole steerable mapping catheter (Livewire, St. Jude Medical Inc, St. Paul, MN, USA) was positioned in the coronary sinus looped around the tricuspid annulus. After transseptal puncture, a 10-pole circular mapping catheter Lasso $^{\mathrm{TM}}$, Biosense Webster, Diamond Bar, CA, USA or Optima ${ }^{\mathrm{TM}}$, St. Jude Medical Inc) was introduced into the left atrium (LA) through an $8 \mathrm{~F}$ transseptal introducer.

\subsubsection{Conventional procedure}

In the CIR group, an irrigated RFA catheter (ThermoCool ${ }^{\circledR}$, Biosense Webster or Navistar ${ }^{\circledR}$ ThermoCool, Biosense Webster) was introduced into the LA through the same puncture site without a second introducer. LA mapping was performed using either Ensite $\mathrm{NavX}^{\mathrm{TM}}$ (St. Jude Medical Inc.) or Carto ${ }^{\mathrm{TM}} \mathrm{XP}$ (Biosense Webster) systems. All 65 patients in the CIR group underwent electrical isolation of all PVs. The ablation consisted of continuous circumferential lesions around each PV, with additional ablation between the two PVs if needed. Twenty-two of these patients underwent PV isolation only. The remainder were given additional ablation of the cavotricuspid isthmus line $(n=9)$, two lines connecting the two contra-lateral superior and inferior veins $(n=7)$ [12], ablation on CFAEs $(n=8)$ or a combination $(n=19)$. The application time of RFA was 40-60 $\mathrm{s}$ at each site and energy was delivered with a cut-off temperature of $50^{\circ} \mathrm{C}$. The maximum output and irrigation rates were $30-35 \mathrm{~W}, 15-20 \mathrm{~mL} / \mathrm{min}$

Table 1 Demographics and procedural data

\begin{tabular}{llll}
\hline & CIR group $(n=65)$ & RMI group $(n=23)$ & RMN group $(n=26)$ \\
\hline Male (\%) & $51(79 \%)$ & $22(96 \%)^{*}$ & $20(77 \%)$ \\
Age (years) ( \pm SD) & $57 \pm 9$ & $59 \pm 7$ & $55 \pm 8$ \\
Paroxysmal AF (\%) & $40(61 \%)$ & $15(65 \%)$ & $14(54 \%)$ \\
Lone AF (\%) & $35(54 \%)$ & $12(52 \%)$ & $13(50 \%)$ \\
Prior AF ablation (\%) & $18(28 \%)$ & $7(30 \%)$ & $6(23 \%)$ \\
PVI without additional ablation & $22(34 \%)$ & $10(43 \%)$ & $6(46 \%)^{\mathrm{a}}$ \\
Procedure time (min) $( \pm$ SD) & $215 \pm 61^{* *}$ & $340 \pm 107$ & $324 \pm 74^{\mathrm{a}}$ \\
Total ablation time (sec) $( \pm$ SD) & $3081 \pm 1515^{* *}$ & $6565 \pm 2206^{* * *}$ & $4737 \pm 1111^{\mathrm{a}}$ \\
Fluoroscopy time (min) $( \pm$ SD) & $46.1 \pm 17$ & $53.7 \pm 22$ & $54.5 \pm 21^{\mathrm{a}}$
\end{tabular}

CIR group conventional manual irrigated catheter; RMI group remote magnetic navigation, irrigated catheter; RMN group remote magnetic navigation, non-irrigated catheter; $P V I$ pulmonary vein isolation; $A F$ atrial fibrillation; $S D$ standard deviation

${ }^{a}$ Only those with PVI confirmed by a circular catheter were included $(n=13)$

*significant vs CIR and RMN, $p<0.05$; ** significant vs RMI and RMN, $p<0.001$; *** significant vs RMN, $p<0.001$ 
(for PV isolation), $25 \mathrm{~W}, 15 \mathrm{~mL} / \mathrm{min}$ in the coronary sinus and $30-40 \mathrm{~W}, 20 \mathrm{~mL} / \mathrm{min}$ for linear or CFAE ablation (maximum $35 \mathrm{~W}$ in $\mathrm{LA}, 40 \mathrm{~W}$ in right atrium (RA)), respectively [13].

\subsubsection{Magnetically guided procedure}

In the patients who underwent $\mathrm{MN}$ ablation, the RFA catheter was introduced into the LA through a second transseptal guiding introducer, and LA mapping was performed by Carto ${ }^{\mathrm{TM}}$ RMT (Biosense Webster). The three-dimensional mapping system was used in conjunction with the Niobe ${ }^{\circledR}$ II system (Stereotaxis, St. Louis, MO, USA). The ablation consisted of a continuous circumferential line around the two ipsilateral PVs, with additional ablation between the two PVs. Energy was applied only when good electrode-tissue contact was indicated by the system monitor. In the RMI group, a Navistar ${ }^{\circledR}$ RMT ThermoCool $^{\circledR}$ (Biosense Webster) ablation catheter was employed. Thirteen patients underwent CFAE ablation and three of these were given additional cavotricuspid isthmus ablation. Application time, output and temperature cut-offs, and irrigation rate were the same as in the CIR group. In the RMN group a Navistar Celsius RMT ${ }^{\mathrm{TM}}$ (Biosense Webster) ablation catheter was used. Eight patients underwent additional CFAE ablation. In 13 patients of this group, the circular mapping catheter was not employed and the PV isolation was confirmed by RFA catheter only [14]. Energy was applied in temperature-controlled mode, with a cut-off temperature of $55^{\circ} \mathrm{C}$ and a maximum output of $40 \mathrm{~W}$. Application time was $20 \mathrm{~s}$ at each site $[1,14]$. Charring of the catheter tip raises its impedance and, if this was suspected, the catheter was extracted and the tip checked out during the procedure.

In all three groups, a Stockert-Cordis (Biosense Webster) RFA generator was utilized. Surface ECG and intracardiac electrograms were recorded utilizing the multichannel LabSystem $^{\mathrm{TM}}$ Pro (Bard Electrophysiology, Lowell, MA, USA). No direct-current cardioversion was performed during the procedures.

\subsection{Blood sampling and marker measurements}

Venous blood samples for baseline data were drawn from an antecubital vein before the procedure in all patients. Post-procedure samples were collected the next morning, $24 \mathrm{~h}$ after the start of the procedure. TnT and CKMB were determined using an electrochemiluminescence immunoassay on a Modular E system (Roche Diagnostics, Mannheim, Germany). The analytical detection limits were $0.01 \mu \mathrm{g} / \mathrm{L}(\mathrm{TnT})$ and $0.1 \mu \mathrm{g} / \mathrm{L}(\mathrm{CKMB})$, respectively. The TnT cut-off value for diagnosis of myocardial infarction according to ACC/AHA guidelines is $0.03 \mu \mathrm{g} / \mathrm{L}$ [15].

\subsection{Follow-up}

Follow-up was performed at the out-patient clinic or by the patients' local hospitals and referring cardiologists. All patients had clinical examination and at least one Holter registration at 3 and 6 months after the procedure and further ECG-recordings if indicated by symptoms. Recurrence was defined as at least one episode of AF lasting more than $60 \mathrm{~s}$ after a blanking period of 3 months. Patients were followed for 1 year after the relevant ablation procedure and clinical results were reported based on this procedure only. Patients who underwent a repeat procedure during the follow-up were therefore regarded as failure, independent of the outcome of the last procedure.

\section{Statistical analyses}

For all statistical analyses, the SPSS software package version 17.0 (SPPS Inc., IL, USA) was used. Discrete variables are reported as counts (percentages) and continuous variables as mean \pm SD. Statistical comparisons were performed using the $\chi^{2}$ for discrete variables and Student's $t$ test, Mann-Whitney $U$ test or one-way ANOVA for continuous variables. Correlations between procedural variables and marker levels were calculated using linear regression analysis and Spearman's rank correlation tests. Levene's test was employed to test the variances between groups. A $p$ value of $<0.05$ was considered to be statistically significant.

\section{Results}

\subsection{Clinical and procedural data}

The demographics and procedural data are shown in Table 1. There were no differences in the distribution of co-morbidity between the three groups. More male patients were included in the RMI group compared to the RMN and CIR groups (96\% vs. $77 \%$ and $79 \%, p<0.01)$. Thirty-one $(27 \%)$ patients had undergone prior AF ablation with an even distribution among the groups. The Procedure time (skin-to-skin) and total ablation time in the RMN group were $276 \pm 76 \mathrm{~min}$ and $3,619 \pm 1,464 \mathrm{~s}$, respectively. In the RMN group, procedure time (232 \pm $46 \min$ vs. $324 \pm 74 \min , p<0.001)$ and total ablation time $(3,194 \pm 853 \mathrm{~s}$ vs. $4,737 \pm 1111 \mathrm{~s}, p<0.001)$ were significantly shorter in patients for whom the circular mapping catheter was not employed for confirmation of $\mathrm{PV}$ isolation. Procedure and total ablation times were longer in the RMI and RMN group than the CIR group $(p<0.001$, Table 1). There were no statistically significant differences 
in fluoroscopy time between the three groups. No procedure-related complications, such as cardiac tamponade, atrioesophageal fistula, PV stenosis, transient ischemic attack or major bleeding were observed in any of the patients.

\subsection{Ablation impact and myocardial markers}

Baseline myocardial marker levels were within the normal range, and displayed a statistically significant increase in both TnT and CKMB after ablation in all groups (Table 2). After ablation there was a significantly lower mean TnT level in the RMN group than in the other groups $(p<0.001)$. If the troponin level was corrected for ablation time $(\mathrm{TnTc})$, the mean $\mathrm{TnTc}(\mu \mathrm{g} / \mathrm{L}$ per 1000 seconds ablation) was considerably higher in the CIR group than in either the RMN or RMI groups (0.61 vs. 0.30 and $0.23 \mu \mathrm{g} / \mathrm{L}, p<0.001)$. The difference in TnTc between the RMN and RMI groups was also statistically significant $(p<0.05)$. Figure 1 shows a greater variance in TnTc level in the CIR group than in either the RMN or RMI groups $(p<0.01)$. There were no differences in any group in the release of $\mathrm{TnTc}$ between patients who underwent PV isolation alone and those who underwent additional LA or RA ablation (CIR group 0.63 and $0.61 \mu \mathrm{g} / \mathrm{L}, p=$ n.s.; RMI group 0.22 and $0.24 \mu \mathrm{g} / \mathrm{L}, p=\mathrm{n} . \mathrm{s}$.; RMN group 0.35 and $0.25 \mu \mathrm{g} / \mathrm{L}, p=0.062$, respectively). In all three groups there were lower levels of TnTc in patients who had undergone prior AF ablation than in those for the first-time procedure (CIR 0.49 vs. $0.67 \mu \mathrm{g} / \mathrm{L}$, $p<0.001$; RMI 0.16 vs. $0.26 \mu \mathrm{g} / \mathrm{L}, p<0.01$; RMN 0.28 vs. $0.36 \mu \mathrm{g} / \mathrm{L}, p<0.01)$. There were no significant differences between the proportions of patients undergoing repeat procedures or ablation additional to $\mathrm{PV}$ isolation in the three groups (Table 1). Within the RMN group, there was a tendency towards higher levels of release of $\mathrm{TnT}$ in patients when a circular mapping catheter was used (1.35 vs. $1.03 \mu \mathrm{g} / \mathrm{L}, p=0.09$ ).

Further analysis revealed a significant correlation between the total ablation time and post-ablation serum concentration of TnT (CIR $r=0.61, p<0.01$; RMI $r=0.74, p<0.001$; and RMN $r=0.51, p<0.01$, respectively; Fig. 2). Post-ablation levels of $\mathrm{CKMB}$ also increased with total ablation time in all groups (CIR $r=0.53, p<0.001$; RMI $r=0.73, p<0.001$; and RMN: $r=0.59, p=0.002$; Fig. 2).

\subsection{Clinical results}

After a mean follow-up period of $12.2 \pm 5.4$ months, 68 patients (59.6\%) were free from $\mathrm{AF}$ recurrence, three patients are still on anti arrhythmic drugs (two patients in CIR group and one in RMI group). Twelve patients used beta blockers because of hypertension or other cardiovascular disease. Longstanding persistent AF patients had significantly lower success rate than other patients $(35.0 \%$ vs. $66.0 \%, p<0.05)$. One patient died during follow-up due to colorectal cancer. There were no differences in success rate in the three different groups (CIR 60.0\%, RMI 61.0\%, RMN 53.8\%, $p=$ n.s.). In the RMN group, there was a significantly higher TnTc in patients with successful ablation compared to patients with recurrence of $\mathrm{AF}(0.43$ vs. $0.25 \mu \mathrm{g} / \mathrm{L}, p<0.01)$, but there were no such differences in the other groups (CIR 0.61 vs. $0.65 \mu \mathrm{g} / \mathrm{L}, p=\mathrm{n} . \mathrm{s}$.; RMI 0.22 vs. $0.24 \mu \mathrm{g} / \mathrm{L}, p=$ n.s.). No differences in total TnT was revealed between patients with or without ablation success in any group.

\section{Discussion}

We measured the levels of myocardial markers TnT and CKMB in patients who underwent manual or remote MN RFA for AF. Previous studies on Troponin I or T have produced diverging results regarding the relationship between enzyme release and the number of RFA lesions and site of RFA [6-10]. Our study demonstrates a significant positive correlation between myocardial marker levels and total ablation time with both manual and $\mathrm{MN}$ catheters.

A considerably lower time-corrected TnT level (TnTc) was associated with the MN system, whether irrigated or non-irrigated catheters were employed. This suggests that myocardial injury, as indicated by enzyme release, was

Table 2 Level of myocardial markers at baseline and after ablation

\begin{tabular}{|c|c|c|c|c|c|c|}
\hline \multirow[t]{2}{*}{ Myocardial marker } & \multicolumn{2}{|c|}{ CIR group } & \multicolumn{2}{|c|}{ RMI group } & \multicolumn{2}{|c|}{ RMN group } \\
\hline & Baseline & After RFA & Baseline & After RFA & Baseline & After RFA \\
\hline $\operatorname{TnT}( \pm \mathrm{SD}) \mu \mathrm{g} / \mathrm{L}$ & $<0.03$ & $1.68 \pm 0.9^{*}$ & $<0.03$ & $1.63 \pm 0.9^{*}$ & $<0.03$ & $1.18 \pm 0.5^{* * * *}$ \\
\hline $\mathrm{CKMB}( \pm \mathrm{SD}) \mu \mathrm{g} / \mathrm{L}$ & $3.4 \pm 1.9$ & $10.1 \pm 4.0^{*}$ & $4.1 \pm 2.3$ & $10.5 \pm 6.3^{*}$ & $3.4 \pm 1.5$ & $10.2 \pm 3.5^{*}$ \\
\hline
\end{tabular}

CIR group conventional manual irrigated catheter; RMI group remote magnetic navigation, irrigated catheter; RMN group remote magnetic navigation, non-irrigated catheter; $R F A$ radiofrequency ablation; $T n T$ troponin T; $C K B M$ creatine kinase's myocardial isoform; $S D$ standard deviation

${ }^{*} p<0.001$, compared to baseline; ${ }^{* *}$ significant vs. RMI and CIR, $p<0.001$ 


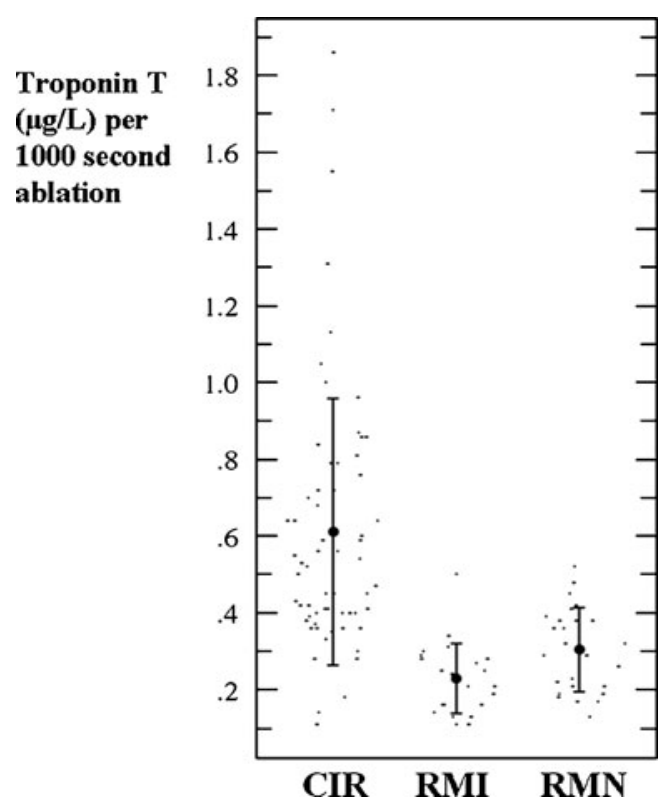

Fig. 1 Scattergrams of ablation time-corrected serum troponin $\mathrm{T}$ level. Mean values with error bars of one standard deviation are shown. Note: there was a larger variance in time-corrected troponin $\mathrm{T}$ in the CIR group than in either the RMI or the RMN groups $(p<0.01)$. $C I R$ conventional manual irrigated catheter, $R M I$ remote magnetic irrigated catheter, $R M N$ remote magnetic non-irrigated catheter lower with the MN system than with the manual ablation technique. Lesions created by MN may appear to be more sharply defined because of the positional stability of the system in comparison with manual catheters, and may therefore cause less myocardial damage and still be effective. It has been suggested that similar mechanisms are involved in cryoablation, when the ablation catheter freezes to the endocardium [16-19].

The manual catheters might be less stable and slide over the endocardium resulting in larger, but shallower and thus less effective, ablation lesions. This lack of stability also might create more inexpedient myocardial damage. AF patients are reported to have a thinner posterior atrial wall [20] and a stable catheter position during ablation may produce transmurality more rapidly than the preset ablation time.

Our results revealed an unexpectedly higher timecorrected release of TnT using a non-irrigated $\mathrm{MN}$ catheter than an irrigated catheter. One possible explanation is that the irrigation flow $(15-20 \mathrm{ml} / \mathrm{min}) \mathrm{might}$ reduce the electrode-tissue contact for the soft catheter. In these two groups there were also different application time, temperature and output cut-off settings, with the highest output and shortest application time in the RMN
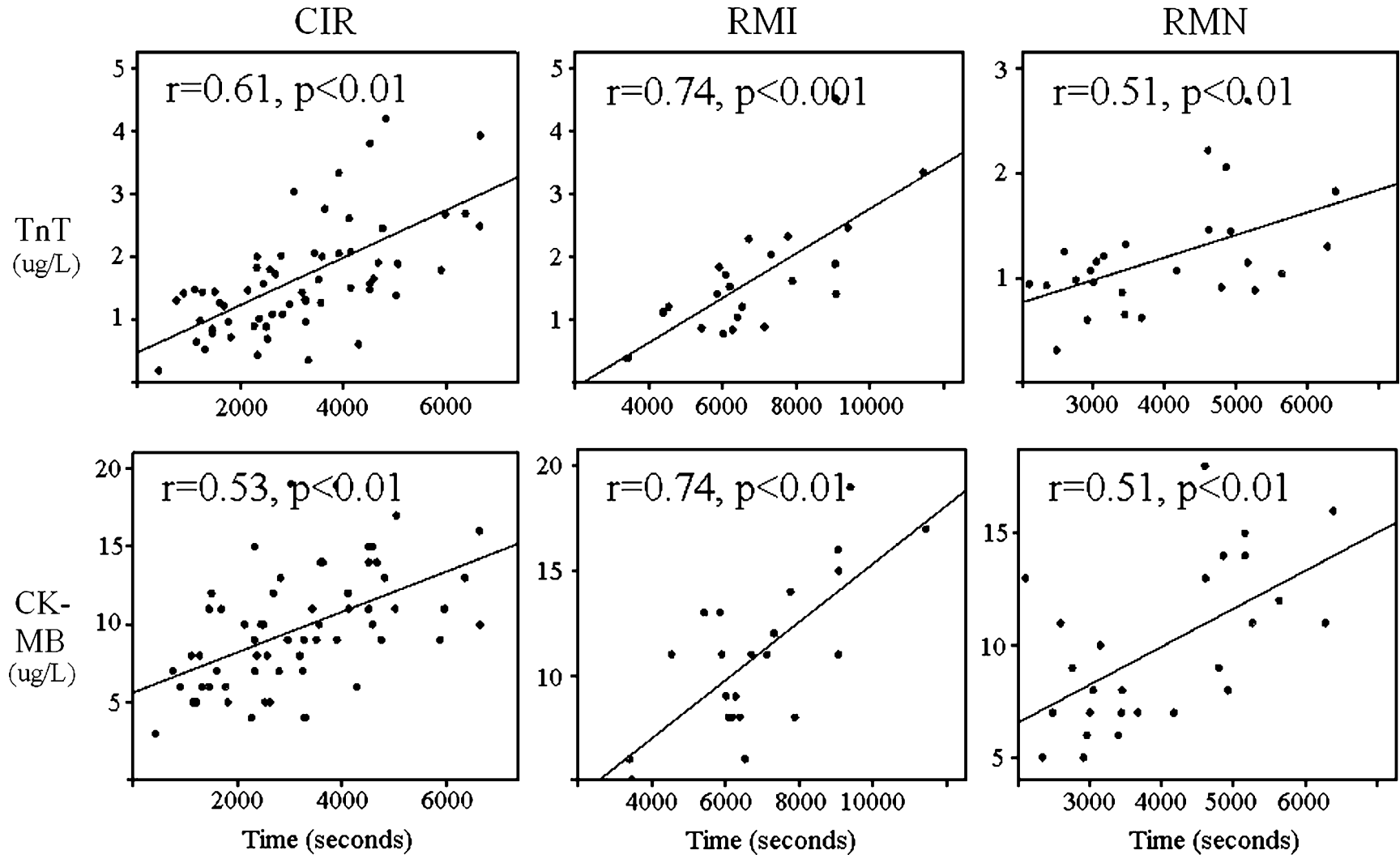

Fig. 2 Correlation of troponin $\mathrm{T}(T n T)$ and cardiac creatin kinase $(C K M B)$ with total ablation time. Upper panels, relationship between serum troponin $\mathrm{T}$ level and total ablation time. Lower panels, relationship between serum CKMB level and total ablation time.

Plots show significant correlations between the myocardial marker levels and total ablation time in all groups. Y-axis serum levels of troponin $\mathrm{T}$ (micrograms per liter) or CKMB (micrograms per liter). $X$-axis total ablation time (seconds). CIR, RMI, RMN; see Fig. 1 
group. The short application time was employed to reduce the risk of char formation [21]. Efficiency is lower during the first few seconds of RFA delivery, until the output reaches a plateau and the lesions might be enlarged up to $180 \mathrm{~s}$ of energy delivery [22, 23]. A longer time to the plateau phase and longer ablation time at each site in the RMI group may have led to a lower TnTc.

On the other hand, the irrigation rate and energy settings were identical in the CIR and RMI groups, and the longer total ablation times in the RMI group did not result in higher levels of myocardial marker release. With the MN system, catheter-tissue contact status can be monitored but no guidance of catheter-tissue contact is yet available with manual catheter ablation. A substantially stronger contact force might create deeper ablation lesions [24, 25]. Our analysis revealed a stronger correlation between $\mathrm{TnT}$ and ablation time with the remote irrigated than the manual irrigated catheter. A possible explanation is that the stable positioning and the low and stable pressure obtained with a remote catheter create more discrete ablation lesions and by that a more predictable ablation effect (Fig. 1). New methods of measuring contact force are being developed, and further studies need to be performed on this subject.

There were no differences in TnTc levels using the MN system between patients who underwent PV isolation alone or who were given additional ablation in the atria. This suggests that catheter movement and tissue contact using remote $\mathrm{MN}$ are similar at the PV ostia and the other atrial locations. A lower level of myocardial marker release was observed in patients who underwent a repeat procedure. Ablation on scars or partly fibrous tissue is not expected to produce a similar release of myocardial markers as ablation on healthy tissue, thus energy was not delivered to areas without electrical signals. Longer procedure and ablation times were needed if a circular mapping catheter was employed to confirm PV isolation (RMN group), supported by the tendency towards higher myocardial marker release in these patients. Since better clinical outcomes have been demonstrated with proven PV isolation [26, 27], it may be necessary to employ a circular mapping catheter also during magnetic navigated AF ablation procedures.

This study was non-randomized, but there were similar baseline characteristics and portions of patients with prior $\mathrm{AF}$ ablation or ablation extensive to PV isolation in the three groups. There were no statistically significant differences in outcome using the different ablation catheters, although there was a tendency to higher AF recurrence rate in the RMN group. Neither were there any differences in marker levels in patients with or without ablation success. In all groups, the proportion of complete PVI and additional tailored ablation on CFAE or complete cavotricuspid isthmus block was similar. This indicates that the choice of procedural strategy is more important than the type of catheter used to reach the endpoints of ablation.

\subsection{Study limitations}

This study was non-randomized which may limit the significance of the outcome on comparisons of the different catheters. The study groups were not identical in size and the numbers of patients in both groups on which the MN system was utilized were relatively small. Although similar proportions of patients underwent individually tailored ablation additional to PV isolation, this approach may limit the interpretation of the clinical results. The temperatures reached and the energy delivered to produce individual lesions was not recorded for comparison. Quantification of electrode-tissue contact and force were not yet an option. The operators' limited experience with the MN system may have had an impact on procedure time, but all operators were experienced electrophysiologists.

\section{Conclusions}

Remote magnetic catheters may create more discrete ablation lesions with a more predictable effect as measured by myocardial enzymes. Employing this system may require longer ablation time to reach the procedural endpoints than manual procedures. Remote magnetic non-irrigated catheters do not appear to be inferior to magnetic irrigated catheters in terms of myocardial marker release and clinical outcome.

Acknowledgments This study was supported by the Norwegian Foundation for Health and Rehabilitation (E Solheim), the Trond Mohn Endowment and the Research Council of Norway.

Open Access This article is distributed under the terms of the Creative Commons Attribution Noncommercial License which permits any noncommercial use, distribution, and reproduction in any medium, provided the original author(s) and source are credited.

\section{References}

1. Di Biase, L., Fahmy, T. S., Patel, D., et al. (2007). Remote magnetic navigation. Human experience in pulmonary vein ablation. Journal of the American College of Cardiology, 50, 868-874.

2. Haissaguerre, M., Jais, P., Shah, D. C., et al. (2000). Electrophysiological end point for catheter ablation of atrial fibrillation initiated from multiple pulmonary venous foci. Circulation, 101, 1409-1417.

3. Pappone, C., Vicedomini, G., Manguso, F., et al. (2006). Robotic magnetic navigation for atrial fibrillation ablation. Journal of the American College of Cardiology, 47, 1390-1400.

4. Thomas, S. P., Aggarwal, G., Boyd, A. C., Jin, Y., \& Ross, D. L. (2004). A comparison of open irrigated and non-irrigated tip catheter ablation for pulmonary vein isolation. Europace, 6, 330-335. 
5. Gerstenfeld, E. P., Jacobson, J., Bazan, V., et al. (2007). Comparison of high power, medium power, and irrigated-tip ablation strategies for pulmonary vein isolation in a canine model. Journal of Cardiovascular Electrophysiology, 18, 849-853.

6. Katritsis, D. G., Hossein-Nia, M., Anastasakis, A., et al. (1997). Use of troponin-T concentration and kinase isoforms for quantification of myocardial injury induced by radiofrequency catheter ablation. European Heart Journal, 18, 1007-1013.

7. Manolis, A. S., Vassilikos, V., Maounis, T., et al. (1999). Detection of myocardial injury during radiofrequency catheter ablation by measuring serum cardiac troponin I levels: procedural correlates. Journal of the American College of Cardiology, 34, 1099-1105.

8. Emkanjoo, Z., Mottadayen, M., Givtaj, N., et al. (2007). Evaluation of post-radiofrequency myocardial injury by measuring cardiac troponin I levels. International Journal of Cardiology, 117, 173-177.

9. Carlsson, J., Erdogan, A., Guettler, N., et al. (2001). Myocardial injury during radiofrequency catheter ablation: comparison of focal and linear lesions. Pacing and Clinical Electrophysiology, 24, 962-968.

10. Hirose, H., Kato, K., Sucuki, O., et al. (2006). Diagnostic accuracy of cardiac markers for myocardial damage after radiofrequency catheter ablation. Journal of Interventional Cardiac Electrophysiology, 16, 169-174.

11. Haegeli, L. M., Kotschet, E., Byrne, J., et al. (2008). Cardiac injury after percutaneous catheter ablation for atrial fibrillation. Europace, 10, 273-275.

12. Chen, J., Off, M. K., Solheim, E., Schuster, P., Hoff, P. I., \& Ohm, O.-J. (2008). Treatment of atrial fibrillation by silencing electrical activity in the posterior inter-pulmonary-vein atrium. Europace, $10,265-272$.

13. Macle, L., Jaïs, P., Weerasooriya, R., et al. (2002). Irrigated-tip catheter ablation of pulmonary veins for treatment of atrial fibrillation. Journal of Cardiovascular Electrophysiology, 13, 1067-1073.

14. Chen, X., Pehrson, S., \& Svendsen, J. H. (2008). Remote magnetic navigation for atrial fibrillation. Heart Rhythm, 5, S171 (Abstract).

15. Anderson, J. L., Adams, C. D., Antman, E. M., et al. (2007). ACC/AHA 2007 guidelines for the management of patients with unstable angina/non-ST-elevation myocardial infarction-executive summary: a report of the American College of Cardiology/ American Heart Association Task Force on Practice Guidelines (Writing Committee to Revise the 2002 Guidelines for the Management of Patients With Unstable Angina/Non-ST-Elevation Myocardial Infarction): developed in collaboration with the American College of Emergency Physicians, American College of Physicians, Society for Academic Emergency Medicine, Society for Cardiovascular Angiography and Interventions, and Society of Thoracic Surgeons. Journal of the American College of Cardiology, 50, 652-726.
16. Katritsis, D., Ellenbogen, K. A., Giazitzoglou, E., et al. (2008). Clinical outcome of atrial fibrillation is related to the extent of radiofrequency ablation. Journal of Interventional Cardiac Electrophysiology, 22, 31-37.

17. Oswald, H., Gardiwal, A., Lissel, C., Tu, H., \& Klein, G. (2007). Difference in humoral biomarkers for myocardial injury and inflammation in radiofrequency ablation versus cryoablation. Pacing and Clinical Electrophysiology, 30, 885-890.

18. Hochcolzer, W., Schlitterhardt, D., Arentz, T., et al. (2007). Platelet activation and myocardial necrosis in patients undergoing radiofrequency and cryoablation of isthmus-dependent atrial flutter. Europace, 9, 490-495.

19. Thornton, A. S., Janse, P., Alings, M., et al. (2008). Acute success and short-term follow-up of catheter ablation of isthmus-depended atrial flutter; a comparisom of $8 \mathrm{~mm}$ tip radiofrequency and cryothermy catheters. Journal of Interventional Cardiac Electrophysiology, 21, 241-248.

20. Platonov, P. G., Ivanov, V., Ho, S. Y., \& Mitrofanova, L. (2008). Left atrial posterior wall thickness in patients with and without atrial fibrillation: Data from 298 consecutive autopsies. Journal of Cardiovascular Electrophysiology, 19, 689-692.

21. Dorwarth, U., Fiek, M., Remp, T., et al. (2003). Radiofrequency catheter ablation: different cooled and noncooled electrode systems induce specific lesion geometries and adverse effects profiles. Pacing and Clinical Electrophysiology, 26, 1438-1445.

22. Hartung, W. M., Burton, M. E., Deam, A. G., \& Walter, P. F. (1995). Mc Teague $\mathrm{K}$ Langberg JJ. Estimation of temperature during radiofrequency catheter ablation using impedance measurements. Pacing and Clinical Electrophysiology, 18, 2017-2021.

23. McRury, I. D., Diamond, S., Falwell, G., Schlichting, A., \& Wilson, C. (2000). The effect of ablation sequence and duration on lesion shape using rapidly pulsed radiofrequency energy through multiple electrodes. Journal of Interventional Cardiac Electrophysiology, 4, 307-320.

24. Eick, O. J., Wittkampf, F. H., Bronneberg, T., \& Schumacher, B. (1998). The LETR-Principle: a novel method to assess electrodetissue contact in radiofrequency ablation. Journal of Cardiovascular Electrophysiology, 9, 1180-1185.

25. Zheng, X., Walcott, G. P., Hall, J. A., et al. (2000). Electrode impedance: an indicator of electrode-tissue contact and lesion dimensions during linear ablation. Journal of Interventional Cardiac Electrophysiology, 4, 645-654.

26. Hocini, M., Sanders, P., Jaïs, P., et al. (2005). Prevalence of pulmonary vein disconnection after anatomical ablation for atrial fibrillation: consequences of wide atrial encircling of the pulmonary veins. European Heart Journal, 26, 696-704.

27. Gerstenfeld, E. P., Callans, D. J., Dixit, S., Zado, E., \& Marchlinski, F. E. (2003). Incidence and location of focal atrial fibrillation triggers in patients undergoing repeat pulmonary vein isolation: implications for ablation strategies. Journal of Cardiovascular Electrophysiology, 14, 691-692. 